Advances in Radio Science (2003) 1: 73-80

(C) Copernicus $\mathrm{GmbH} 2003$

\title{
Kontinuierliche und diskrete Differenzialformen als Ausgangspunkt für numerische Methoden in der Elektrodynamik
}

\author{
S. Kurz \\ Robert Bosch GmbH, FV/FLO, P.O. Box 106050, 70049 Stuttgart, Germany
}

Zusammenfassung. Die Grundgleichungen der Elektrodynamik werden häufig in integraler Form aufgestellt. Die Umwandlung in partielle Differenzialgleichungen geschieht dann durch Anwendung der Integralsätze von Gauss und Stokes. Beide Integralsätze besitzen eine große formale Ähnlichkeit. Formuliert man die Maxwellschen Gleichungen mit Hilfe von Differenzialformen, wird diese zunächst formale Analogie verständlich, als Konsequenz eines abstrakteren Konzeptes. Neben der damit einhergehenden übersichtlichen und eleganten Darstellung der Elektrodynamik erhält man einen gut geeigneten Ausgangspunkt für numerische Methoden.

Differenzialformen besitzen natürliche Entsprechungen im Diskreten, die diskreten Differenzialformen. Das hieraus in niedrigster Ordnung resultierende Diskretisierungsschema entspricht der Allokation von Freiheitsgraden auf zueinander dualen Gittersystemen, wie sie von der FIT (= Finite Integration Technique) bekannt ist.

Größere Freiheiten hat man bei der Diskretisierung der Materialbeziehungen, die auf diskrete Hodge-Operatoren führt. Je nach verwendetem Ansatz (orthogonale oder baryzentrische duale Gitter) erhält man unterschiedliche numerische Verfahren. Kontinuierliche und diskrete Differenzialformen können deshalb als allgemeiner Ausgangspunkt für numerische Methoden in der Elektrodynamik betrachtet werden.

The fundamental laws of electrodynamics are often stated in integral form. The conversion to partial differential equations is conveyed by application of the integral theorems of Gauss and Stokes. Both theorems bear a strong formal resemblance. If Maxwell's equations are reformulated in terms of differential forms this seemingly formal analogy will become obvious, as a consequence of a more abstract underlying concept. Besides the accompanying concise and elegant formulation of electrodynamics a useful starting point for numerical methods is obtained.

Correspondence to: S. Kurz (stefan.kurz@gmx.de)
Differential forms possess natural correspondents in the discrete setting, the so called discrete differential forms. The resulting discretization scheme of lowest order corresponds to the allocation of degerees of freedom on dual grid pairs, which is well known from the FIT (= Finite Integration Technique).

There is more freedom when the constitutive equations are to be discretized, which yields discrete Hodge operators. Depending on the employed technique (orthogonal or barycentric dual grids) one ends up with different numerical schemes. Continuous and discrete differential forms can therefore be regarded as a general starting point for numerical methods in electrodynamics.

\section{Grundlegende Eigenschaften von Differenzialformen}

Differenzialformen (DF) können völlig unabhängig von irgendwelchen Koordinatensystemen als Felder von Multikovektoren definiert werden. Anschaulicher ist es jedoch, DF als vollständige Integranden anzusehen, wie sie unter einem Integralzeichen erscheinen (Deschamps, 1981; Flanders, 1989). Der Einfachheit halber beschränken wir uns hier auf DF im dreidimensionalen Euklidisch-affinen Raum und kartesische Koordinaten $\boldsymbol{x}=(x, y, z)$. Dann haben wir von den drei linear unabhängigen Differenzialen ersten Grades $\mathrm{d} x, \mathrm{~d} y, \mathrm{~d} z$ auszugehen. Eine DF ersten Grades oder Pfaff'sche Form, kurz 1-Form, entsteht daraus durch Linearkombination mit Koeffizientenfunktionen,

$\omega_{1}=f(\boldsymbol{x}) \mathrm{d} x+g(\boldsymbol{x}) \mathrm{d} y+h(\boldsymbol{x}) \mathrm{d} z$.

Von besonderer Bedeutung ist der Fall, dass die Koeffizientenfunktionen gerade mit den Koordinaten $\left(a_{x}, a_{y}, a_{z}\right)$ eines Vektorfeldes identifiziert werden. Man spricht dann von einer dem Vektorfeld $\boldsymbol{a}$ zugeordneten 1-Form und schreibt dafür

${ }^{1} \boldsymbol{a}=a_{x} \mathrm{~d} x+a_{y} \mathrm{~d} y+a_{z} \mathrm{~d} z$.

Differenziale werden mit Hilfe des äußeren Produktes $\wedge$ multipliziert. Das äußere Produkt verhält sich assoziativ und 
distributiv wie das gewöhnliche Produkt, und alternierend wie das Kreuzprodukt, das heißt die Reihenfolge der Faktoren ist erheblich,

$\mathrm{d} x \wedge \mathrm{d} y=-\mathrm{d} y \wedge \mathrm{d} x, \quad \mathrm{~d} x \wedge \mathrm{d} x=0$.

Von den neun möglichen Produkten der drei Differenziale ersten Grades sind nur drei linear unabhängig, die drei Differenziale zweiten Grades $\mathrm{d} y \wedge \mathrm{d} z, \mathrm{~d} z \wedge \mathrm{d} x, \mathrm{~d} x \wedge \mathrm{d} y$. Durch Linearkombination mit Koeffizientenfunktionen kann man 2Formen bilden,

$\omega_{2}=f(\boldsymbol{x}) \mathrm{d} y \wedge \mathrm{d} z+g(\boldsymbol{x}) \mathrm{d} z \wedge \mathrm{d} x+h(\boldsymbol{x}) \mathrm{d} x \wedge \mathrm{d} y$.

Mit ${ }^{2} \boldsymbol{b}=b_{x} \mathrm{~d} y \wedge \mathrm{d} z+b_{y} \mathrm{~d} z \wedge \mathrm{d} x+b_{z} \mathrm{~d} x \wedge \mathrm{d} y$ ist die dem Vektorfeld $\boldsymbol{b}=\left(b_{x}, b_{y}, b_{z}\right)$ zugeordnete 2-Form gemeint, man beachte die zyklische Abfolge der Koordinaten.

Schließlich gibt es ein linear unabhängiges Differenzial dritten Grades, $\mathrm{d} x \wedge \mathrm{d} y \wedge \mathrm{d} z$, mit den zugehörigen 3-Formen

$\omega_{3}=g(\boldsymbol{x}) \mathrm{d} x \wedge \mathrm{d} y \wedge \mathrm{d} z={ }^{3} g$

Hier wurde ein skalares Feld $g$ als Koeffizientenfeld verwendet, was auf die 3-Form ${ }^{3} g$ führt. Der Vollständigkeit halber sei noch gesagt, dass skalare Felder für sich genommen als 0-Formen aufgefasst werden können.

Geht man zu anderen Koordinaten über, indem man zum Beispiel $x=x(u, v), y=y(u, v)$ setzt, ergibt sich das korrekte Transformationsverhalten der Differenziale ganz automatisch aus der alternierenden Eigenschaft des äußeren Produktes, denn es ist

$$
\begin{aligned}
\mathrm{d} x & \wedge \mathrm{d} y=\left(\frac{\partial x}{\partial u} \mathrm{~d} u+\frac{\partial x}{\partial v} \mathrm{~d} v\right) \wedge\left(\frac{\partial y}{\partial u} \mathrm{~d} u+\frac{\partial y}{\partial v} \mathrm{~d} v\right) \\
& =\left(\frac{\partial x}{\partial u} \frac{\partial y}{\partial v}-\frac{\partial x}{\partial v} \frac{\partial y}{\partial u}\right) \mathrm{d} u \wedge \mathrm{d} v \\
& =\frac{\partial(x, y)}{\partial(u, v)} \mathrm{d} u \wedge \mathrm{d} v
\end{aligned}
$$

mit der Funktionaldeterminante $\frac{\partial(x, y)}{\partial(u, v)}$. Analog erhält man $\mathrm{d} x \wedge \mathrm{d} y \wedge \mathrm{d} z=\frac{\partial(x, y, z)}{\partial(u, v, w)} \mathrm{d} u \wedge \mathrm{d} v \wedge \mathrm{d} w$.

Unter gewissen recht allgemeinen Voraussetzungen, die die Existenz der Integrale sicherstellen, kann eine $p$-Form $\omega$ über ein $p$-dimensionales Gebiet $\Omega(p=1$ : Linie, $p=2$ : Fläche, $p=3$ : räumliches Gebiet) integriert werden. Dazu geht man von einer Parameterdarstellung des Integrationsgebietes aus und rechnet in einem ersten Schritt die DF in den Parameterraum um, so wie in Gl. 1. Das Parameterdifferenzial wird mit dem Linien-, Flächen- oder Volumenelement identifiziert, so dass man ein gewöhnliches (mehrfaches) Integral bekommt. Integriert man eine 1-Form entlang einer Linie (Paramter $t$ ), erhält man

$$
\begin{aligned}
\int_{C}{ }_{C} \boldsymbol{a} & =\int_{C} a_{x} \mathrm{~d} x+a_{y} \mathrm{~d} y+a_{z} \mathrm{~d} z \\
= & \int_{t_{1}}^{t_{2}}\left(a_{x} \frac{\partial x}{\partial t}+a_{y} \frac{\partial y}{\partial t}+a_{z} \frac{\partial z}{\partial t}\right) \mathrm{d} t=\int_{C} \boldsymbol{a} \cdot \mathrm{d} \boldsymbol{C},
\end{aligned}
$$

mit dem Linienelement $\mathrm{d} \boldsymbol{C}=\frac{\partial \boldsymbol{x}}{\partial t} \mathrm{~d} t=\left(\frac{\partial x}{\partial t}, \frac{\partial y}{\partial t}, \frac{\partial z}{\partial t}\right) \mathrm{d} t$. Das ist gerade das aus der Vektoranalysis bekannte Arbeitsintegral. Die Integration einer 2-Form über eine Fläche (Parameter $u, v$ ) führt auf

$$
\begin{aligned}
\int_{F}^{2} \boldsymbol{b} & =\int_{F} b_{x} \mathrm{~d} y \wedge \mathrm{d} z+b_{y} \mathrm{~d} z \wedge \mathrm{d} x+b_{z} \mathrm{~d} x \wedge \mathrm{d} y \\
& =\int_{u_{1}}^{u_{2}} \int_{v_{1}}^{v_{2}}\left(b_{x} \frac{\partial(y, z)}{\partial(u, v)}+b_{y} \frac{\partial(z, x)}{\partial(u, v)}+b_{z} \frac{\partial(x, y)}{\partial(u, v)}\right) \\
\underbrace{\mathrm{d} u}_{=+\mathrm{d} u \mathrm{~d} v} \wedge \mathrm{d} v & \int_{F} \boldsymbol{b} \cdot \mathrm{d} \boldsymbol{F}
\end{aligned}
$$

mit dem vektoriellen Flächenelement $\mathrm{d} \boldsymbol{F}=\left(\frac{\partial \boldsymbol{x}}{\partial u} \times \frac{\partial \boldsymbol{x}}{\partial v}\right) \mathrm{d} u \wedge$ $\mathrm{d} v=\left(\frac{\partial(y, z)}{\partial(u, v)}, \frac{\partial(z, x)}{\partial(u, v)}, \frac{\partial(x, y)}{\partial(u, v)}\right) \mathrm{d} u \mathrm{~d} v$. Gleichung 3 gibt den Fluss des Vektorfeldes $\boldsymbol{b}$ durch die Fläche $F$. Durch die Reihenfolge der Parameter wird eine Orientierung des Integrationsgebietes festgelegt, was sich in Gl. 3 durch die Festlegung $\mathrm{d} u \wedge \mathrm{d} v=+\mathrm{d} u \mathrm{~d} v$ niederschlägt. Eine Vertauschung der Parameter $u$ und $v$ hätte einen Vorzeichenwechsel des Integrals zur Folge. Für das Integral $I$ einer $p$-Form $\omega$ über ein $p$-dimensionales Gebiet $\Omega$ schreiben wir im folgenden kurz $I=\omega \mid \Omega$.

DF können nicht nur integriert, sondern auch differenziert werden. Die Differenziation von DF verallgemeinert die Bildung des Differenzials $\mathrm{d} f$ einer skalaren Funktion $f$. Allgemein wird durch den linearen Differenzialoperator d einer $p$-Form $\omega$ eine $(p+1)$-Form $\mathrm{d} \omega$ zugeordnet, die äußere oder Cartansche Ableitung genannt wird. Man kann das durch folgenden formalen Ausdruck beschreiben

$\mathrm{d}=\mathrm{d} x \wedge \frac{\partial}{\partial x}+\mathrm{d} y \wedge \frac{\partial}{\partial y}+\mathrm{d} z \wedge \frac{\partial}{\partial z}$,

das heißt das Argument wird partiell nach einer Koordinate differenziert, wobei Differenziale wie Konstanten zu behandeln sind. Anschließend wird von links das entsprechende Koordinatendifferenzial anmultipliziert. Der größte Nutzen des Differenzialoperators d liegt darin, dass mit ihm die Operatoren grad, rot und div der Vektoranalysis zusammengefasst und verallgemeinert werden können. Man rechnet leicht nach, dass

$\mathrm{d}^{0} f={ }^{1}(\operatorname{grad} f)$,

$\mathrm{d}^{1} \boldsymbol{a}=^{2}(\operatorname{rot} \boldsymbol{a})$,

$\mathrm{d}^{2} \boldsymbol{b}={ }^{3}(\operatorname{div} \boldsymbol{b})$,

$\mathrm{d}^{3} g=0$ gilt.

Die vielleicht wichtigste Eigenschaft des Differenzialoperators ist $\mathrm{d}(\mathrm{d} \omega)=0$, das sog. Lemma von Poincaré (Flanders, 1989; Schleifer, 1983). Das ist nichts anderes als die Gleichheit der gemischten partiellen Ableitungen der Koeffizientenfunktionen. Setzt man $\omega={ }^{0} f$ findet man ${ }^{2}(\operatorname{rot} \operatorname{grad} f)=0$, und aus $\omega={ }^{1} \boldsymbol{a}$ folgt ${ }^{3}(\operatorname{div} \operatorname{rot} \boldsymbol{a})=0$. Die Wirbelfreiheit von Gradientenfeldern und die Quellenfreiheit von Wirbelfeldern sind demnach Konsequenzen des Lemmas von Poincaré. 
Tabelle 1. Integralsätze der Vektoranalysis als Spezialfälle des allgemeinen Integralsatzes von Stokes

\begin{tabular}{cccccccccccc}
\hline Integralsatz & \multicolumn{2}{c}{$\mathrm{d} \omega$} & $\Omega$ & \multicolumn{7}{c}{$\Gamma=\partial \Omega$} \\
\hline Hauptsatz & 1-Form & ${ }^{1}(\operatorname{grad} f)$ & Kurve & $C$ & 0-Form & ${ }^{0} f$ & Endpunkte & $\partial C$ & $\int_{C}(\operatorname{grad} f) \cdot \mathrm{d} \boldsymbol{C}$ & $=f\left(\boldsymbol{x}_{2}\right)-f\left(\boldsymbol{x}_{1}\right)$ \\
Satz von Stokes & 2-Form & ${ }^{2}(\operatorname{rot} \boldsymbol{a})$ & Fläche & $F$ & 1-Form & ${ }^{1} \boldsymbol{a}$ & Randkurve & $\partial F$ & $\int_{F}(\operatorname{rot} \boldsymbol{a}) \mathrm{d} \boldsymbol{F}$ & $=\oint_{\partial F} \boldsymbol{a} \cdot \mathrm{d} \boldsymbol{C}$ \\
Satz von Gauss & 3-Form & ${ }^{3}(\operatorname{div} \boldsymbol{b})$ & Gebiet & $V$ & 2-Form & ${ }^{2} \boldsymbol{b}$ & Oberfläche & $\partial V$ & $\int_{V} \operatorname{div} \boldsymbol{b} \mathrm{d} V$ & $=\oint_{\partial V} \boldsymbol{b} \cdot \mathrm{d} \boldsymbol{F}$ \\
\hline
\end{tabular}

Tabelle 2. Zuordnung von DF zu den Feldern und Potentialen der Elektrodynamik

\begin{tabular}{llllllllll}
\hline DF & \multicolumn{1}{c}{ Bezeichung, physikalische Einheit } & DF & & Bezeichung, physikalische Einheit \\
\hline$\varphi=$ & ${ }^{0} \varphi$ & 0-Form & Skalares elektrisches Potential & in V & $\underline{E}=$ & ${ }^{1} \boldsymbol{E}$ & 1-Form & Elektrische Feldstärke & in V \\
$\underline{A}=$ & ${ }^{1} \boldsymbol{A}$ & 1-Form & Magnetisches Vektorpotential & in Vs & $\underline{B}=$ & ${ }^{2} \boldsymbol{B}$ & 2-Form & Magnetische Induktion & in Vs \\
$\underline{j}=$ & ${ }^{2} \boldsymbol{j}$ & 2-Form & Elektrische Stromdichte & in A & $\underline{H}=$ & ${ }^{1} \boldsymbol{H}$ & 1-Form & Magnetische Feldstärke & in A \\
$\underline{\underline{\rho}}=$ & ${ }^{3} \rho$ & 3-Form & Elektrische Raumladung & in As & $\underline{D}=$ & ${ }^{2} \boldsymbol{D}$ & 2-Form & Dielektrische Verschiebung & in As \\
\hline
\end{tabular}

Der Zusammenhang zwischen der Differenziation und der Integration wird durch den allgemeinen Integralsatz von Stokes hergestellt. Er kann mit Hilfe von DF besonders elegant ausgedrückt werden. Es sei $\Omega$ ein $(p+1)$-dimensionales orientiertes Gebiet mit dazu konsistent orientiertem Rand $\Gamma=\partial \Omega$, und $\omega$ eine in einer Umgebung von $\Omega \cup \Gamma$ stetig differenzierbare DF. Dann gilt $\mathrm{d} \omega|\Omega=\omega| \partial \Omega$. Alle bekannten Integralsätze der Vektoranalysis, die Integrale über ein Gebiet und Integrale über dessen Rand miteinander in Beziehung setzen, sind darin als Spezialfälle enthalten, siehe Tabelle 1 .

\section{Formulierung der Maxwellschen Gleichungen mit Differenzialformen}

DF erlauben eine besonders übersichtliche und elegante Darstellung der Elektrodynamik (Burke, 1985; Deschamps, 1981; Schleifer, 1983; Warnick et al., 1997; Wyler, 1967). Man geht am besten von den Maxwellschen Gleichungen in integraler Form aus. Der Grad der DF ergibt sich aus der Dimension des jeweiligen Integrationsgebietes.

Auf diese Weise entstehen die in Tabelle 2 getroffenen $\mathrm{Zu}$ ordnungen. Mit Hilfe dieser Zuordnungen und der Beziehungen aus Abschnitt 1 rechnet man leicht nach, dass die nachstehende Formulierung der Maxwellschen Gleichungen mit DF korrekt ist,

$$
\begin{aligned}
& \left.\begin{array}{l}
\operatorname{rot} \boldsymbol{E}=-\frac{\partial \boldsymbol{B}}{\partial t} \Leftrightarrow \mathrm{d} \underline{E}=-\frac{\partial \underline{B}}{\partial t} \Leftrightarrow\left[D^{1}\right]\{\underline{E}\}=-\frac{\partial\{\underline{B}\}}{\partial t}, \\
\operatorname{div} \boldsymbol{B}=0 \Leftrightarrow\left[D^{2}\right]\{\underline{B}\}=0,
\end{array}\right\} \\
& \left.\begin{array}{rl}
\operatorname{rot} \boldsymbol{H}=\boldsymbol{j}+\frac{\partial \boldsymbol{D}}{\partial t} \Leftrightarrow \mathrm{d} \underline{H}=\underline{j}+\frac{\partial \underline{D}}{\partial t} \Leftrightarrow\left[\tilde{D}^{1}\right]\{\underline{\tilde{H}}\}=\{\underline{\tilde{j}}\}+\frac{\partial\{\underline{\tilde{D}}\}}{\partial t}, \\
\operatorname{div} \boldsymbol{D}=\rho \quad \Leftrightarrow \mathrm{d} \underline{D}=\underline{\rho} \quad \Leftrightarrow\left[\tilde{D}^{2}\right]\{\underline{\tilde{D}}\}=\{\underline{\tilde{\rho}}\} .
\end{array}\right\}
\end{aligned}
$$

Hierbei wurden die üblichen Bezeichnungen für die Felder verwendet. Der Gleichungssatz in der rechten Spalte beschreibt eine diskrete Darstellung der Maxwellschen Gleichungen, die im nächsten Abschnitt besprochen wird.

\section{Diskrete Differenzialformen}

DF besitzen natürliche Entsprechungen im Diskreten, die diskreten DF. Um zu diskreten DF zu gelangen, muss das Rechengebiet in einzelne Elemente unterteilt werden, zum Beispiel in Tetraeder. In dem hierdurch erzeugten Gitter $\mathcal{T}_{h}$ kann man geometrische Objekte unterschiedlicher Dimension identifizieren, siehe Abb. 1 links. Die orientierten Knoten, Kanten, Flächen und Zellen werden unter dem Oberbegriff der Facetten zusammengefasst. Die Menge aller orientierten $p$-Facetten $\mathcal{S}_{p}=\left\{s_{i}, i=1, \ldots, S_{p}\right\}$ enthält $S_{p}$ Elemente.

Die nachfolgenden Betrachtungen sind dabei keineswegs auf Tetraederelemente beschränkt. Es kommen genauso gut andere Elementtypen in Frage, solange ein reguläres FENetz (FE = Finite Elemente) erhalten bleibt. Auf jeden Fall wird vorausgesetzt, dass sich der Rand einer $(p+1)$-Facette $s_{i} \in \mathcal{S}_{p+1}$ durch $p$-Facetten $b_{j} \in \mathcal{S}_{p}$ in der Form 


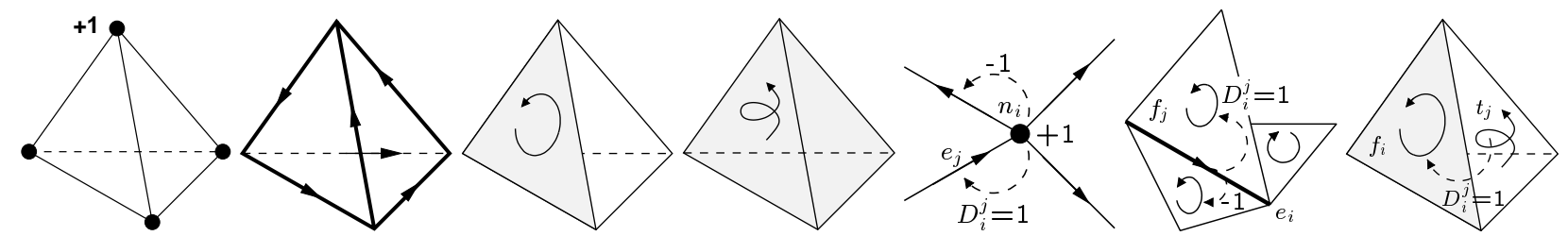

Abbildung 1. Links: Beispiele für orientierte Knoten $n$, Kanten $e$, Flächen $f$ und Zellen $t$. Rechts: Relative Orientierungen von Knoten und Kanten, Kanten und Flächen, sowie Flächen und Zellen.

$\partial s_{i}=\sum_{j=1}^{S_{p}} D_{i}^{j} b_{j}, D_{i}^{j}=\left\{\begin{array}{c}0 \text { Facette } b_{j} \text { nicht im Rand } \partial s_{i} \text { enthalten, } \\ \pm 1 \text { Facette } b_{j} \text { im Rand } \partial s_{i} \text { enthalten, mit relativer Orientierung } \pm 1\end{array}\right.$

darstellen lässt. Mit relativer Orientierung +1 ist gemeint, dass die Facette $b_{j}$ konsistent orientiert ist bezüglich der Orientierung der Facette $s_{i}$. Beispiele für unterschiedliche relative Orientierungen sind in Abb. 1 rechts dargestellt.
Grundgedanke bei der Diskretisierung von DF ist nun, dass nicht mehr alle denkbaren Integrationsgebiete zugelassen werden, sondern nur noch jene, die sich aus $p$-Facetten $s_{i} \in \mathcal{S}_{p}$ des Gitters zusammensetzen lassen, sog. $p$-Ketten ${ }^{1}$

$\Omega=\sum_{i=1}^{S_{p}} \Omega^{i} s_{i}, \quad \Omega^{i}=\left\{\begin{array}{r}0 \text { Facette } s_{i} \text { nicht in der Kette } \Omega \text { enthalten, } \\ 1 \text { Facette } s_{i} \text { in der Kette } \Omega \text { enthalten, in Richtung der Orientierung }, \\ -1 \text { Facette } s_{i} \text { in der Kette } \Omega \text { enthalten entgegen der Orientierung. }\end{array}\right.$

Die Koeffizienten $\Omega^{i}$ kann man zu einem Koeffizientenvektor $\{\Omega\}=\left(\Omega^{1}, \Omega^{2}, \ldots, \Omega^{S_{p}}\right)^{\mathrm{T}}$ zusammenfassen. Integriert man eine $p$-Form $\omega$ über eine $p$-Kette $\Omega$, findet man

$\omega \mid \Omega=\sum_{i=1}^{S_{p}}\left(\omega \mid s_{i}\right) \Omega^{i}=\sum_{i=1}^{S_{p}} \omega_{i} \Omega^{i}$

mit $\omega_{i}=\omega \mid s_{i}$.

Um den Wert des Integrals zu berechnen, braucht man die kontinuierliche DF nicht vollständig zu kennen. Es genügt, wenn die $S_{p}$ Koeffizienten $\omega_{i}$ bekannt sind, die ebenfalls zu einem Koeffizientenvektor $\{\omega\}=\left(\omega_{1}, \omega_{2}, \ldots, \omega_{S_{p}}\right)^{\mathrm{T}} \mathrm{zu}-$ sammengefasst werden. DF vermitteln lineare Abbildungen vom Typ Integrationsgebiet $\rightarrow$ reelle Zahl. Eine kontinuierliche DF ordnet jedem Integrationsgebiet eine reelle Zahl zu, während eine diskrete DF nur den auf dem Gitter darstellbaren Integrationsgebieten eine reelle Zahl zuordnet. Die diskrete DF wird durch den Koeffizientenvektor $\{\omega\}$ und das Integrationsgebiet durch den Koeffizientenvektor $\{\Omega\}$ repräsentiert. An die Stelle des Integrals $\omega \mid \Omega$ tritt nun die Berechnungsvorschrift

$\{\omega\} \mid\{\Omega\}:=\sum_{i=1}^{S_{p}} \omega_{i} \Omega^{i}$.

Die Diskretisierung der kontinuierlichen DF hat dadurch zu geschehen, dass die Integrale $\omega_{i}=\omega \mid s_{i}$ berechnet werden. Das bedeutet für eine 0-Form die Bestimmung der Werte an den Knoten, für eine 1-Form des Arbeitsintegrals entlang der Kanten, für eine 2-Form der Flüsse durch die Flächen und für eine 3-Form der Inhalte in den Zellen. Insoweit stimmt dieses
Diskretisierungsverfahren mit der Allokation von Freiheitsgraden bei der FIT (=Finite Integration Technique) überein (Weiland, 1996).

Schreibt man Gl. 9 für eine $(p+1)$-Kette an und wendet darauf den Randoperator $\partial$ an, bekommt man mit Gl. 8

$$
\begin{aligned}
& \partial \Omega=\sum_{i=1}^{S_{p+1}} \Omega^{i} \partial s_{i}=\sum_{i=1}^{S_{p+1}} \sum_{j=1}^{S_{p}} \Omega^{i} D_{i}^{j} b_{j} \\
& =\sum_{j=1}^{S_{p}}(\partial \Omega)^{j} b_{j} \text { mit } \quad(\partial \Omega)^{j}=\sum_{i=1}^{S_{p+1}} D_{i}^{j} \Omega^{i} .
\end{aligned}
$$

Der Randoperator $\partial$ besitzt die diskrete Repräsentation

$$
\begin{aligned}
& \{\partial \Omega\}=\left[D^{p}\right]^{\mathrm{T}}\{\Omega\} \\
& {\left[D^{p}\right]=\left(\begin{array}{ccc}
D_{1}^{1} & \cdots & D_{1}^{S_{p}} \\
\vdots & & \vdots \\
D_{S_{p+1}}^{1} & \cdots & D_{S_{p+1}}^{S_{p}}
\end{array}\right) \in \mathbf{R}^{S_{p+1} \times S_{p}} .}
\end{aligned}
$$

Die Matrix $\left[D^{p}\right]$, die lediglich von der Topologie des Gitters abhängt, wird auch als Inzidenzmatrix zwischen $p$ - und $(p+1)$-Facetten bezeichnet. Sie spielt die Rolle einer diskreten äußeren Ableitung, wie man durch Diskretisierung des allgemeinen Integralsatzes von Stokes, $\mathrm{d} \omega|\Omega=\omega| \partial \Omega$, nachweisen kann,

\footnotetext{
${ }^{1}$ Die allgemeine Definition von Ketten geht von reellen Koeffizienten aus.
} 


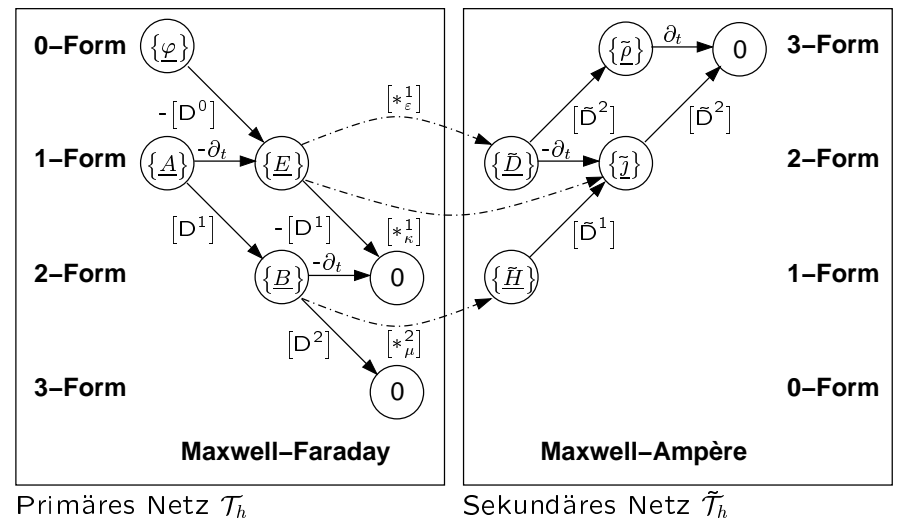

Abbildung 2. Darstellung der diskretisierten Maxwellschen Gleichungen, der Definitionsgleichungen der Potentiale, der Kontinuitätsgleichung und der Materialbeziehungen als Tonti-Diagramm. Die im Kreis stehende Größe ergibt sich als Summe der auf den Kreis zulaufenden durchgezogenen Pfeile. Die durch die Materialbeziehungen gegebenen Relationen sind mit gestrichelten Pfeilen dargestellt (Tonti).

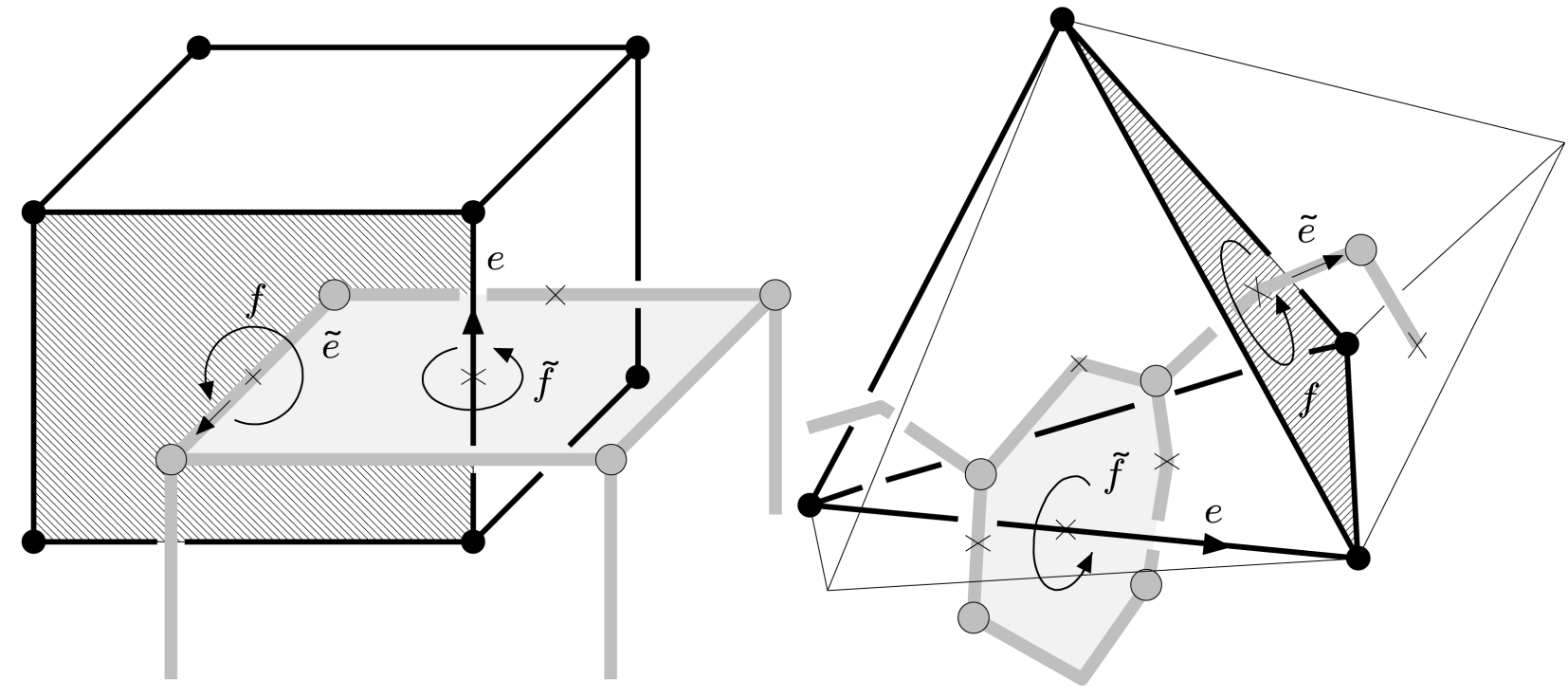

Abbildung 3. Primale Gitter (schwarz) mit zugehörigen dualen Gittern (grau). Die primale Kante $e$ korrespondiert mit der dualen Fläche $\tilde{f}$, die primale Fläche $f$ korrespondiert mit der dualen Kante $\tilde{e}$. Dabei ergänzen sich die Orientierungen der Kante und der Fläche zur Raumorientierung. Links: Orthogonales duales Gitter, ausgehend von einem primalen Hexaedergitter. Rechts: Baryzentrisches duales Gitter, ausgehend von einem primalen Tetraedergitter (Bossavit, 1999b).

$$
\begin{aligned}
& \{\mathrm{d} \omega\}|\{\Omega\}=\{\omega\}|\{\partial \Omega\}=\{\omega\}\left|\left[D^{p}\right]^{T}\{\Omega\}=\left[D^{p}\right]\{\omega\}\right|\{\Omega\} \\
& \{\mathrm{d} \omega\}=\left[D^{p}\right]\{\omega\} .
\end{aligned}
$$

Anwendung des Differenzialoperators d auf eine kontinuierliche $p$-Form entspricht also Multiplikation des Koeffizientenvektors der zugehörigen diskreten DF mit der Matrix $\left[D^{p}\right]$. Mit dieser Korrespondenz kann man eine diskrete Fassung der Maxwellschen Gleichungen anschreiben, was in der rechten Spalte der Gln. 6 und 7 geschehen ist. Dabei sind die Koeffizientenvektoren noch von der Zeit abhängig. Es handelt sich also um ein System gewöhnlicher Differenzialgleichungen erster Ordnung in der Zeit.

Die Gln. 6 und 7 sind nur über die Materialbeziehungen miteinander gekoppelt. Das bedeutet, dass sie auf voneinander unabhängigen Gittern diskretisiert werden können. Wir bezeichnen diese beiden Gitter mit $\mathcal{T}_{h}$ (primäres Gitter) und $\tilde{\mathcal{T}}_{h}$ (sekundäres Gitter). Alle dem sekundären Gitter $\tilde{\mathcal{T}}_{h}$ zuge- ordneten Größen werden mit einer Tilde gekennzeichnet, so wie in Gl. 7. Die Freiheit bei der Wahl von $\mathcal{T}_{h}$ und $\tilde{\mathcal{T}}_{h}$ kann dazu verwendet werden, um zu einer geschickten Diskretisierung der Materialbeziehungen zu gelangen.

\section{Diskretisierung der Materialbeziehungen}

Es ist klar, dass die Materialbeziehungen bei der Verwendung von DF selbst im einfachsten Fall nicht durch skalare Faktoren beschrieben werden können, da DF unterschiedlichen Grades miteinander verknüpft werden müssen. In der klassischen Theorie der DF wird der Hodge-Operator $*$ eingeführt, der $p$-Formen auf $(n-p)$-Formen abbildet, $n$ ist die Dimension des Raumes. Man kann ihn im dreidimensionalen Euklidisch-affinen Raum auf folgende Weise definieren,

$$
*_{\alpha}\left\{\begin{array}{c}
1 \rightarrow \alpha^{3} \mathrm{~d} x \wedge \mathrm{d} y \wedge \mathrm{d} z \rightarrow 1 \\
\mathrm{~d} x \rightarrow \alpha \mathrm{d} y \wedge \mathrm{d} z \rightarrow \mathrm{d} x
\end{array}\right.
$$


mit einem Materialparameter $\alpha>0$. Die übrigen Fälle entstehen durch zyklische Vertauschung der Raumkoordinaten. Offenbar ist $*_{\alpha}\left(*_{\alpha} \omega\right)=\omega$ und aus Gl. 15 folgt $*_{\alpha}^{0} f=$ $\alpha^{3}\left({ }^{3} f\right), *_{\alpha}^{1} \boldsymbol{a}=\alpha\left({ }^{2} \boldsymbol{a}\right), *_{\alpha}^{2} \boldsymbol{b}=\alpha^{-1}\left({ }^{1} \boldsymbol{b}\right)$ und $*_{\alpha}^{3} g=\alpha^{-3}\left({ }^{0} g\right)$. Mit Hilfe dieses Operators kann man die Materialbeziehungen folgendermaßen aufschreiben,

$$
\begin{gathered}
\boldsymbol{D}=\varepsilon \boldsymbol{E} \Leftrightarrow \underline{D}=*_{\varepsilon} \underline{E} \Leftrightarrow\{\underline{\tilde{D}}\}=\left[*_{\varepsilon}^{1}\right]\{\underline{E}\}, \\
\boldsymbol{H}=\frac{1}{\mu} \boldsymbol{B} \Leftrightarrow \underline{H}=*_{\mu} \underline{B} \Leftrightarrow\{\underline{\tilde{H}}\}=\left[*_{\mu}^{2}\right]\{\underline{B}\}, \\
\boldsymbol{j}=\kappa \boldsymbol{E} \Leftrightarrow \underline{j}=*_{\kappa} \underline{E} \Leftrightarrow\{\underline{\tilde{j}}\}=\left[*_{\kappa}^{1}\right]\{\underline{E}\},
\end{gathered}
$$

hierin bedeuten $\varepsilon$ die Dielektrizitätszahl (DZ), $\mu$ die Permeabilität und $\kappa$ die el. Leitfähigkeit. In der rechten Spalte wurde eine diskrete Fassung der Materialbeziehungen angegeben. Die Frage nach einer Diskretisierung der Materialbeziehungen ist gleichwertig mit der Frage nach diskreten HodgeOperatoren. Diskrete Hodge-Operatoren werden durch Matrizen repräsentiert, der hochgestellte Index kennzeichnet jeweils den Grad der diskreten DF, auf die der Operator wirkt.

Die diskreten Grundgleichungen der Elektrodynamik lassen sich übersichtlich in Form eines formalen Ablaufdiagramms, des sog. Tonti-Diagramms darstellen, siehe Abb. 2. Diagonale Pfeile stehen für räumliche, horizontale Pfeile für zeitliche Ableitungen. Die in einem Kreis stehende Größe ergibt sich als Summe der auf den Kreis zulaufenden durchgezogenen Pfeile. Die Bildung der äußeren Ableitung behält die physikalische Dimension einer DF bei. Deshalb besitzen im Diagramm alle Größen, die auf einer Diagonale stehen, dieselbe Dimension. Die gestrichelten Pfeile repräsentieren Materialbeziehungen.

Wie man in Abb. 2 gut erkennen kann, ist der Zusammenhang zwischen den diskreten DF auf dem primären und dem sekundären Gitter durch diskrete Hodge-Operatoren gegeben, deren Konstruktion nun genauer betrachtet werden soll.

Wir beschränken uns hier auf solche diskrete HodgeOperatoren, die durch quadratische Matrizen dargestellt werden können. Dazu ist es erforderlich, dass die Anzahl der $p$-Facetten von $\mathcal{T}_{h}$ mit der Anzahl der $(n-p)$-Facetten von $\tilde{\mathcal{T}}_{h}$ übereinstimmt, $S_{p}=\tilde{S}_{n-p}$. Das kann erreicht werden, indem man duale Gitter verwendet. Man bezeichnet in diesem Fall $\mathcal{T}_{h}$ als primales und $\tilde{\mathcal{T}}_{h}$ als duales Gitter. $\mathrm{Zu}$ jeder primalen $p$-Facette $s_{i}$ existiert eine eindeutig bestimmte duale $(n-p)$ Facette $\tilde{s}_{i}$, die mit der primalen Facette genau einen Punkt gemeinsam hat. Die Nummerierung wird so gewählt, dass korrespondierende primale und duale Facetten denselben Index besitzen. Zwei Familien dualer Gitter sind von besonderem Interesse und in Abb. 3 dargestellt, nämlich orthogonale und baryzentrische duale Gitter.

Um numerisch stabile und physikalisch sinnvolle Diskretisierungen zu erhalten, müssen die Matrizen der diskreten Hodge-Operatoren symmetrisch und positiv definit vorausgesetzt werden. Weiterhin ist Konsistenz eine notwendige Bedingung für Konvergenz bei der Verfeinerung der Gitter. Mit Konsistenz ist gemeint, dass die diskrete Fassung der Materialgesetze mit der kontinuierlichen Form verträglich sein muss. Betrachten wir ein Paar zusammengehöriger Felder $(\underline{D}, \underline{E})$, für die $\underline{D}=*_{\varepsilon} \underline{E}$ gilt. Das diskretisierte Paar $(\{\underline{\tilde{D}}\},\{\underline{E}\})$ soll dann über den diskreten Hodge-Operator zusammenhängen, $\underline{\tilde{D}}=\left[*_{\varepsilon}^{1}\right]\{\underline{E}\}$. Es ist klar, dass diese Forderung nicht für ganz beliebige Felder zu erfüllen ist. Mindestens für homogene Felder soll die Forderung nach Konsistenz aber exakt erfüllt sein. Betrachten wir dazu ein homogenes Feld $\underline{D}$ und ein Material mit konstanter DZ $\varepsilon$, so dass $\underline{E}$ ebenfalls homogen ist. Der Fluss von $\underline{D}$ durch die duale Fläche $\tilde{f}_{i}$ lässt sich in der Form $\{\underline{\tilde{D}}\}_{i}=\underline{D} \mid \tilde{f}_{i}=\boldsymbol{D} \cdot \tilde{\boldsymbol{f}}_{i}$ darstellen. Für ebene Flächen ist $\tilde{f}_{i}$ der Flächennormalenvektor, sein Betrag ist gleich dem Flächeninhalt $\left\|\tilde{f}_{i}\right\|$. Für das Arbeitsintegral von $\underline{E}$ entlang der primalen Kante $e_{j}$ findet man analog $\{\underline{E}\}_{j}=\underline{E} \mid e_{j}=\boldsymbol{E} \cdot \boldsymbol{e}_{j}$. Für geradlinige Kanten ist $\boldsymbol{e}_{j}$ ein Vektor in Richtung der Kante, dessen Betrag mit der Länge $\left\|e_{j}\right\|$ der Kante übereinstimmt. Die Forderung nach Konsistenz für beliebige homogene Felder $\underline{D}$ führt auf $\boldsymbol{D} \cdot \tilde{\boldsymbol{f}}_{i}=\sum_{j=1}^{E}\left[*_{\varepsilon}^{1}\right]^{i j} \boldsymbol{D} \cdot \boldsymbol{e}_{j} / \varepsilon(E=$ Zahl der primalen Kanten), das heißt auf das geometrische Konsistenzkriterium

$\tilde{\boldsymbol{f}}_{i}=\sum_{j=1}^{E}\left[*_{\varepsilon}^{1}\right]^{i j} \frac{1}{\varepsilon} \boldsymbol{e}_{j}$.

Die hierbei getroffene Annahme homogener Felder lässt sich anschaulich dadurch rechtfertigen, dass auf einer geeignet gewählten räumlichen Skala jedes glatte Feld in guter Näherung lokal durch ein Homogenfeld beschrieben werden kann. Eine problemangepasste Diskretisierung muss so beschaffen sein, dass sie diese Skala widerspiegelt, um lokal richtige Aussagen liefern zu können. Der Materialparameter $\varepsilon$ wird im allgemeinen Fall jedoch nicht räumlich konstant sein. Man geht dann von einer auf die primalen Zellen bezogenen, stückweise konstanten Materialverteilung und einem stückweise homogenen el. Feld aus. In Gl. 19 ist dann ein effektiver Materialparameter $\bar{\varepsilon}$ einzusetzen, der sich auf geeignete Weise aus den Werten der betroffenen primalen Zellen berechnet (Bossavit, 1999b).

Im folgenden beschränken wir uns auf solche Gitter, deren Zellen Polyeder sind.

\subsection{Orthogonale duale Gitter}

Orthogonale duale Gitter sind dadurch gekennzeichnet, dass sich korrespondierende Kanten und Flächen rechtwinklig schneiden, wie in Abb. 3 links. In diesem Fall lässt sich eine besonders einfache Konstruktion der diskreten HodgeOperatoren angeben. Aufgrund der Orthogonalität sind die Vektoren $\tilde{\boldsymbol{f}}_{i}$ und $\boldsymbol{e}_{i}$ parallel, und es ist $\tilde{\boldsymbol{f}}_{i}=\left(\left\|\tilde{f}_{i}\right\| /\left\|e_{i}\right\|\right) \boldsymbol{e}_{i}$. Man erkennt, dass unter dieser Voraussetzung das Kriterium 19 durch die Wahl von $\left[*_{\varepsilon}^{1}\right]^{i j}=\left(\varepsilon\left\|\tilde{f}_{i}\right\| /\left\|e_{i}\right\|\right) \delta^{i j}$ erfüllt werden kann. Ähnliche Überlegungen für diskrete $p$-Formen führen auf den diagonalen, positiv definiten diskreten HodgeOperator

$\left[*_{\alpha}^{p}\right]^{i j}=\alpha^{3-2 p} \frac{\left\|\tilde{s}_{i}\right\|}{\left\|s_{j}\right\|} \delta^{i j}, \quad \tilde{s}_{i} \in \tilde{\mathcal{S}}_{3-p}$,

$s_{j} \in \mathcal{S}_{p}, \quad i, j=1, \ldots, S_{p}$. 
Die Koeffizienten des diskreten Hodge-Operators 20 enthalten somit Informationen über die Metrik des Gitters (Längen-, Flächen-, Rauminhalte) sowie über den Materialparameter. Der durch Gl. 20 beschriebene diskrete HodgeOperator stimmt für $p=1,2$ genau mit den Materialmatrizen der FIT überein (Clemens und Weiland, 2001). Anders gesagt: Die FIT kann als eine Diskretisierung der Maxwellschen Gleichungen mit diskreten DF auf orthogonalen dualen Gittern mit diskreten Hodge-Operatoren nach Eq. (20) aufgefasst werden.

Im Falle eines uniformen primalen Hexaedergitters lässt sich das orthogonale duale Gitter sehr leicht durch Verschiebung konstruieren, vgl. Abb. 3 links. Im allgemeinen ist es aber nicht einfach und zuweilen gar nicht möglich, zu einem beliebigen primalen Gitter ein orthogonales duales Gitter zu finden. Aus diesem Grunde ist es wünschenswert, eine Konstruktionsverfahren für diskrete Hodge-Operatoren zur Hand zu haben, welches nicht auf der Orthogonalität der Gitter beruht. Falls die Gitter nicht orthogonal sind, lässt sich das Kriterium 19 jedoch im allgemeinen nicht mit diagonalen diskreten Hodge-Operatoren erfüllen.

\subsection{Baryzentrisches duales Gitter}

Geht man zum Beispiel von einem primalen Tetraedergitter aus, so wie in Abb. 3 rechts, kann man ein baryzentrisches duales Gitter verwenden. Das baryzentrische duale Gitter ist ein Sonderfall einer allgemeineren Konstruktion, die für sternförmige Zellen anwendbar ist (Bossavit, 1999a). Die dualen Knoten sind die Schwerpunkte (Baryzenter) der primalen Zellen. Duale Kanten entstehen, indem duale Knoten mit den Schwerpunkten der primalen Flächen verbunden werden. Aus dualen Kanten lassen sich duale Flächen und daraus duale Zellen bilden.

Tetraeder besitzen den Vorteil, dass für sie eine systematische Interpolationsvorschrift für diskrete DF existiert. Dabei handelt es sich um lokale Basis- $p$-Formen $\beta_{p}^{j}(\boldsymbol{x})$, die sog. Whitney-Formen (Bossavit, 2000a; Whitney, 1957). Sie besitzen die Interpolationseigenschaft $\beta_{p}^{j}(\boldsymbol{x}) \mid s_{i}=\delta_{i}^{j}$ mit $s_{i} \in \mathcal{S}_{p}\left(i, j=1, \ldots, S_{p}\right)$, und verallgemeinern deshalb die bekannten Lagrangeschen Ansatzfunktionen für knotenbasierte finite Elemente $(p=0)$. Mit Hilfe der WhitneyFormen lässt sich eine kontinuierliche interpolierende DF $\omega_{h}(\boldsymbol{x})$ aus der diskreten DF $\{\omega\}$ gewinnen,

$\omega_{h}(\boldsymbol{x})=\sum_{j=1}^{S_{p}} \omega_{j} \beta_{p}^{j}(\boldsymbol{x})=\{\omega\}^{\mathrm{T}}\left\{\beta_{p}(\boldsymbol{x})\right\}$

mit $\left\{\beta_{p}(\boldsymbol{x})\right\}=\left(\beta_{p}^{1}(\boldsymbol{x}), \beta_{p}^{2}(\boldsymbol{x}), \ldots, \beta_{p}^{S_{p}}(\boldsymbol{x})\right)^{\mathrm{T}}$.

Whitney-Formen besitzen die fundamentale Eigenschaft

$\mathrm{d}\left\{\beta_{p}(\boldsymbol{x})\right\}=\left[D^{p}\right]^{\mathrm{T}}\left\{\beta_{p+1}(\boldsymbol{x})\right\}, \quad$ mithin

$\mathrm{d} \omega_{h}(\boldsymbol{x})=\left(\left[D^{p}\right]\{\omega\}\right)^{\mathrm{T}}\left\{\beta_{p+1}(\boldsymbol{x})\right\}$,

das heißt kontinuierliche und diskrete äußere Ableitung dürfen vertauscht werden. Das hat eine äußerst interessante und merkwürdige Konsequenz: Interpoliert man eine Lösung der diskreten Maxwellschen Gleichungen mit
Whitney-Formen, erhält man eine exakte Lösung der kontinuierlichen Maxwellschen Gleichungen. Die Betrachtungsweise mit DF zeigt, dass der gesamte Diskretisierungsfehler bei der Diskretisierung der Materialbeziehungen entsteht. Es genügt, Konvergenz- und Fehlerbetrachtungen auf die diskreten Hodge-Operatoren zu beziehen (Hiptmair, 2001).

Bossavit (2000b) hat gezeigt, dass sich unter den gegebenen Voraussetzungen (primales Tetraedergitter, baryzentrisches duales Gitter, bezüglich der primalen Zellen stückweise konstante Materialeigenschaften) ein diskreter HodgeOperator, der das Kriterium 19 erfüllt, aus den WhitneyFormen folgendermaßen berechnen lässt,

$\left[*_{\alpha}^{p}\right]^{i j}=\left(\alpha^{3-2 p} \beta_{p}^{i}(\boldsymbol{x}) \cdot \beta_{p}^{j}(\boldsymbol{x}) \mathrm{d} U\right) \mid U, i, j=1, \ldots, S_{p}$.

Dabei ist $U$ das diskretisierte Gebiet und $\mathrm{d} U$ das Volumenelement der Euklidischen Metrik, eine 3-Form. Das Skalarprodukt zweier $p$-Formen stimmt mit jenem der entsprechenden Felder überein. Gleichung 23 definiert einen symmetrischen, positiv definiten Hodge-Operator, den sog. "GalerkinHodge“" Zur Verdeutlichung soll Gl. 23 für den oben betrachteten Fall $\alpha=\varepsilon, p=1$ angeschrieben werden,

$\left[*_{\varepsilon}^{1}\right]^{i j}=\left(\varepsilon \beta_{1}^{i}(\boldsymbol{x}) \cdot \beta_{1}^{j}(\boldsymbol{x}) \mathrm{d} U\right) \mid U=\int_{U} \varepsilon \boldsymbol{b}_{1}^{i}(\boldsymbol{x}) \cdot \boldsymbol{b}_{1}^{j}(\boldsymbol{x}) \mathrm{d} U(24)$

mit $\beta_{1}^{i}={ }^{1}\left(\boldsymbol{b}_{1}^{i}\right)$ Die den Whitney-1-Formen zugeordneten Basis-Vektorfelder $\boldsymbol{b}_{1}^{i}(\boldsymbol{x})$ sind nichts anderes als die Ansatzfunktionen für kantenbasierte finite Elemente. Die Matrix 24 wird in der Theorie der finiten Elemente als Massenmatrix bezeichnet.

Um den Zusammenhang zur FE-Methode noch deutlicher zu machen, betrachten wir als einfachstes Beispiel die Elektrostatik. Die zu lösende Gleichung lautet mit Operatoren der Vektoranalysis, mit kontinuierlichen und mit diskreten DF ausgedrückt

$$
\begin{aligned}
\operatorname{div} \varepsilon \operatorname{grad} \varphi & =-\rho \Leftrightarrow \mathrm{d} *_{\varepsilon} \mathrm{d} \underline{\varphi} \\
=-\underline{\rho} & \Leftrightarrow\left[\tilde{D}^{2}\right]\left[*_{\varepsilon}^{1}\right]\left[D^{0}\right]\{\underline{\varphi}\}=-\{\underline{\tilde{\rho}}\} .
\end{aligned}
$$

Aufgrund der Dualität der Gitter $\mathcal{T}_{h}$ und $\tilde{\mathcal{T}}_{h}$ lassen sich die dualen durch primale Inzidenzmatrizen ausdrücken. In Abb. 3 links kann man beispielsweise erkennen, dass $e$ und $f$ konsistent orientiert sind, Inzidenz +1 . Das hat für das duale Gitter zur Folge, dass auch $\tilde{e}$ und $\tilde{f}$ konsistent orientiert sind. Ähnliche Überlegungen kann man für die anderen $p$-Facetten anstellen. Man findet allgemein $\left[\tilde{D}^{2-p}\right]=$ $(-1)^{p+1}\left[D^{p}\right](p=0, \ldots, 3)$, im vorliegend Fall also $\left[\tilde{D}^{2}\right]=$ $-\left[D^{0}\right]$. Wir setzen deshalb $[K]=\left[D^{0}\right]\left[*_{\varepsilon}^{1}\right]\left[D^{0}\right]$ und haben $[K]\{\varphi\}=\{\tilde{\rho}\}$ zu lösen. Wegen der fundamentalen Eigenschaft 22 der Whitney-Formen kann man die diskreten Ableitungen in der Definition von $[K]$ durch kontinuierliche Ableitungen ausdrücken. Ausgehend von Gl. 24 findet man für die Elemente der Matrix

$$
\begin{aligned}
& {[K]^{i j}=\left(\varepsilon \mathrm{d} \beta_{0}^{i}(\boldsymbol{x}) \cdot \mathrm{d} \beta_{0}^{j}(\boldsymbol{x}) \mathrm{d} U\right) \mid U} \\
& =\int_{U} \varepsilon \operatorname{grad} b_{0}^{i}(\boldsymbol{x}) \cdot \operatorname{grad} b_{0}^{j}(\boldsymbol{x}) \mathrm{d} U, \quad i, j=1, \ldots, N .
\end{aligned}
$$


Dabei sind die $b_{0}^{i}(\boldsymbol{x})$ gerade die $N$ Ansatzfunktionen für knotenbasierte finite Elemente, $[K]$ ist die zugehörige Steifigkeitsmatrix. Schließlich kann man zeigen, dass für die Koeffizienten der rechten Seite gilt

$\{\underline{\tilde{\rho}}\}_{i}=\int_{U} b_{0}^{i}(\boldsymbol{x}) \rho_{h}(\boldsymbol{x}) \mathrm{d} U, \quad i=1, \ldots, N$.

Mit $\rho_{h}(\boldsymbol{x})=\sum_{i=1}^{N} \rho\left(\boldsymbol{x}_{i}\right) b_{0}^{i}(\boldsymbol{x})$ ist eine knotenbasierte Interpolante der gegebenen Raumladungsdichte $\rho$ (x) gemeint. Abgesehen von der speziellen Diskretisierung der rechten Seite ergeben sich genau dieselben Gleichungen wie bei Anwendung der Standard-Galerkin-FE-Methode. Insbesondere sind in den Gln. 26 und 27 keinerlei Bezüge zum dualen Gitter mehr enthalten. Die Diskretisierung der Maxwellschen Gleichungen mit diskreten DF unter Verwendung des "Galerkin-Hodge"-Operators 23 führt nach Elimination der dualen Größen auf nichts anderes als die Standard-GalerkinFE-Methode. Umgekehrt kann die FIT als ein GalerkinVerfahren mit perturbierten Massenmatrizen aufgefasst werden, was für theoretische Zwecke äußerst nützlich ist, wie Hiptmair (2001) gezeigt hat.

Der Vollständigkeit halber sei noch erwähnt, dass Whitney-Formen nicht nur für Tetraeder, sondern auch für eine Reihe anderer Zellgeometrien konstruierbar sind (Gradinaru und Hiptmair, 1999).

\section{Literatur}

Bossavit, A., Computational electromagnetism and geometry: Building a finite-dimensional „Maxwell's house“. (1): Network equations, J. Japan Soc. Appl. Electromagn. \& Mech., 7, 150-159, 1999a.

Bossavit, A., Computational electromagnetism and geometry. (2): Network constitutive laws, J. Japan Soc. Appl. Electromagn. \& Mech., 7, 294-301, 1999b.
Bossavit, A., Computational electromagnetism and geometry. (4): From degrees of freedom to fields, J. Japan Soc. Appl. Electromagn. \& Mech., 8, 102-109, 2000a.

Bossavit, A., Computational electromagnetism and geometry. (5): The „Galerkin hodge“, J. Japan Soc. Appl. Electromagn. \& Mech., 8, 203-209, 2000b.

Burke, W., Applied differential geometry, Cambridge University Press, Cambridge, 1985.

Clemens, M. und Weiland, T., Discrete electromagnetism with the finite integration technique, in Geometric Methods for Computational Electromagnetics, herausgegeben von F. Teixeira, Bd. 32 von Progress in Electromagnetics Research, S. 65-87, EMW Publishing, Cambridge, Massachusetts, 2001.

Deschamps, G., Electromagnetics and differential forms, Proceedings of the IEEE, 69, 676-696, 1981.

Flanders, H., Differential Forms, Dover Publications, New York, 1989.

Gradinaru, V. und Hiptmair, R., Whitney elements on pyramids, Techn. Ber. 113, SFB 382, Universität Tübingen, 1999.

Hiptmair, R., Discrete Hodge operators: an algebraic perspective, in Geometric Methods for Computational Electromagnetics, herausgegeben von F. Teixeira, Bd. 32 von Progress in Electromagnetics Research, S. 247-269, EMW Publishing, Cambridge, Massachusetts, 2001.

Schleifer, N., Differential forms as a basis for vector analysis - with applications to electrodynamics, American Journal of Physics, 51, 1139-1145, 1983.

Tonti, E., Discrete physics, http://www.dic.univ.trieste.it/perspage/tonti/.

Warnick, K., Selfridge, R., und Arnold, D., Teaching electromagnetic field theory using differential forms, IEEE Transactions on Education, 40, 53-68, 1997.

Weiland, T., Time domain electromagnetic field computation with finite difference methods, International Journal of Numerical Modelling: Electronic Networks, Devices and Fields, 9, 259319, 1996.

Whitney, H., Geometric Integration Theory, Princeton University Press, Princeton, New Jersey, USA, 1957.

Wyler, O., Exterior differential calculus and Maxwell's equations, in Lectures on Calculus, herausgegeben von K. May, S. 147-165, Holden-Day, San Francisco, CA, 1967. 